\title{
EDITORIAL
}

\section{Pragmatic prognostic approach of rheumatoid arthritis- associated interstitial lung disease}

\author{
V. Cottin ${ }^{*} \#$
}

$\mathbf{R}$ heumatoid arthritis (RA) affects approximately $1 \%$ of the adult population, with female predominance. Pulmonary involvement is common, and may include a variety of manifestations namely RA-associated interstitial lung disease (ILD) [1, 2], pleural disease [3], rheumatoid nodules and airway complications (crico-arytenoid arthritis, bronchiectasis, bronchiolitis [4]). ILD is usually detected in patients already diagnosed with RA (mostly between the ages of 50 and 60 yrs and more commonly in males), but isolated pulmonary disease may precede the onset of articular disease. Clinically significant RA-ILD affects approximately $7 \%$ of patients with RA [5], whereas a higher prevalence was found by autopsy studies or prospective screening studies using high-resolution chest tomography (HRCT) [1]. Thus, RA-ILD may affect up to one out of 1,000 adult persons in the general population. When present, it may decrease the survival of patients by 3.5 to $4.9 \mathrm{yrs}$ [6], although the outcome is highly variable, with indolent course and conversely rapid deterioration being reported. Tobacco smoking, a potentially reversible risk factor, increases the incidence and severity of RA [7] through a proposed mechanism of epitope citrullination in a predisposing genetic background [8], increases the risk of severe extra-articular manifestations of RA [5] and might increase the risk of having ILD in patients with RA although conflicting results have been reported $[1,9]$.

Much of the complexity of interstitial pneumonia arises from the lack of a definite relationship between histopathological and imaging phenotypes. The currently available data indicate that the histopathological pattern of usual interstitial pneumonia (UIP) may be equally as frequent as non-specific interstitial pneumonia (NSIP) in patients with RA-ILD [10], contrasting with the predominance of NSIP in the other connective tissue diseases. Patterns of organising pneumonia, lymphocytic interstitial pneumonia, diffuse alveolar damage, and even desquamative interstitial pneumonia have also been observed. However, UIP in connective tissue disease may not exactly replicate idiopathic UIP (which defines idiopathic pulmonary fibrosis (IPF)), with fewer fibroblastic foci [11, 12], smaller honeycombing spaces, and more pronounced inflammation and germinal centres [12]. In addition, the interstitial pneumonia in RA is frequently associated with lymphocytic bronchiolitis [4],

*Hospices civils de Lyon, Hôpital Louis Pradel, Service de pneumologie - centre de référence des maladies pulmonaires rares, " Université de Lyon, Université Lyon I, Lyon, France.

CORRESPONDENCE: V. Cottin, Hôpital Louis Pradel, 69677 Lyon (Bron) Cedex, France. E-mail: vincent.cottin@chu-lyon.fr with well-developed inducible bronchus-associated lymphoid tissue likely involved in the pathogenesis [13].

Studies of the prognosis of ILD in connective tissue disease are few and give conflicting results. The largest study of wellphenotyped patients supports the hypothesis of a better survival of patients with RA-ILD (and generally of ILD in connective tissue disease) than IPF [14]. However, the subgroup of patients with RA and a histopathological pattern of UIP had a similar outcome as those with IPF/UIP [14]. Such studies, however, suffer from major referral and selection bias, because patients with RA-ILD infrequently undergo surgical lung biopsy (due to frequent comorbidities and to the apparent lack of correlation between histopathology and response to therapy). Series of patients with RA-ILD and available histopathology include cases with atypical imaging features referred to tertiary centres and are unlikely to represent the broad population of affected patients. Although a trend was found toward worse survival of RA-ILD with UIP histological pattern as compared to those with a NSIP pattern [14], it is uncertain whether published series are representative of the often stable disease [15] frequently encountered in the routine care of patients with mild RA-ILD.

With this in mind, one may wonder whether we actually need to know the histopathological pattern in RA-ILD; does it really matter? In other words, it may be hypothesised that the extent or pattern of disease on HRCT and serial changes in pulmonary physiology may be sufficient to predict mortality, as in IPF [16-18]. In addition, significant progress has been made in identifying imaging features that are predictive of the histopathological pattern of UIP $[19,20]$ or NSIP [21]. In IPF, a typical "imaging pattern of UIP" on HRCT, a terminology that will need refinement to avoid confusion between imaging and pathological patterns, is predictive of the histopathological pattern of UIP and may obviate the need of lung biopsy. However in RA-ILD, pathological (HRCT correlation) studies are scarce and include a limited number of patients [22]. The accuracy of the imaging pattern is thus not well known in RAILD, and it is presently uncertain that the imaging pattern predicts outcome in RA-ILD.

In the current issue of the European Respiratory Journal, KIM et al. [23] report on a retrospective series of 82 patients with RA-ILD. They sought to determine whether an imaging pattern suggestive of UIP had prognostic significance in RA-ILD. Specific HRCT features were reviewed independently in a blinded fashion by two radiologists, and the pattern on HRCT was classified as "definite UIP" defined as basilar predominant 
reticulation, traction bronchiectasis and honeycombing, with limited ground-glass abnormality; "likely NSIP", defined as predominant bibasilar ground-glass attenuation with limited or no reticulation and absent honeycombing; or "indeterminate UIP/NSIP" pattern. Using this imaging classification, a "definite UIP pattern on HRCT" was found in $24 \%$ of patients and a "likely NSIP pattern" in $23 \%$. Two important observations were made in this study. First, the survival of patients with RA-ILD was clearly associated with the imaging pattern (with a poorer outcome in those with "definite UIP pattern" on HRCT). Second, the survival of patients with RA-ILD and "definite UIP pattern on HRCT" was similar to those of control patients with a clinical radiological diagnosis of IPF (using the same HRCT criteria), thus demonstrating that the imaging pattern is clinically relevant in RA-ILD. Specific features associated with a poor outcome were traction bronchiectasis, honeycomb fibrosis, male sex and lower baseline carbon monoxide diffusing capacity.

The main originality of the article by KIM et al. [23] is that histopathological patterns were not taken into account into the survival analysis, with both RA-UIP and IPF groups studied based on the same HRCT criteria. Outcome was studied with regard to the imaging pattern, regardless of the histopathology. Actually, a minority of patients had lung biopsy performed, with poor correlation between histopathology and imaging features. If these observations are confirmed, such imaging-based approaches could be routinely applicable to evaluate prognosis in patients with RA-ILD.

The study has several limitations. The lack of surgical lung biopsy in a majority of patients precludes pathological radiological correlations. More importantly, the causes of death were not evaluated in this study, an important limitation given the non-respiratory mortality of patients with RA [24]. Emphysema when associated to infiltrative features may significantly alter our ability to distinguish honeycomb lung from emphysematous changes with associated reticulation [25], a factor that was not taken into account in the present study. The definition of the "UIP" and "likely NSIP" patterns on imaging is mostly an inference from what has been shown in idiopathic interstitial pneumonias, but there is only partial evidence that this holds true in the context of RA-ILD. Although the study contributes to predict the prognosis of patients with a "UIP" or "likely NSIP" pattern on imaging, we are left with little information for more than half of the patients who have "indeterminate UIP/NSIP" pattern on imaging.

The article by KIM et al. [23] significantly contributes to a rational and pragmatic approach of patients with RA-ILD, filling some of the gaps in our knowledge. It is likely that histopathological assessment is needed in only a minority of patients with RA-ILD, with most medical decisions being made on the basis of non-invasive evaluation. In contrast with idiopathic interstitial pneumonias (where the surgical lung biopsy is invaluable when the imaging pattern is not typical of IPF), the histopathology may be dispensable to assess for prognosis and to predict response (or lack of response) to therapy in RA-ILD.

A number of questions remain unexplored. Does the extent of disease on imaging predict long-term survival as shown in systemic sclerosis [26], possibly beyond the dichotomous UIP/ non-UIP pattern classification? Does the duration of rheumatological disease and/or of RA-ILD affect the imaging pattern? Is the imaging pattern modified by tobacco smoking? Does the imaging pattern affect the response to therapy and the risk/ benefit ratio of treatment? Additional studies are also needed to appreciate whether RA-ILD may be affected by therapy given for the rheumatic disease (e.g. methotrexate); indeed, despite isolated reports of improvement of RA-ILD by antitumour necrosis factor- $\alpha$ agents, there is a growing body of literature suggesting that these agents can exacerbate the pulmonary disease (in addition to increasing the incidence of pulmonary infections) and should be used with caution in patients with pre-existing RA-ILD [27].

Despite its prevalence and prognostic significance, RA-ILD has not been the focus of much attention in recent years. Prospective studies exploring the diagnostic and the therapeutic approaches are eagerly awaited. At present, the careful analysis of HRCT features can help us to better inform our patients about their disease and potential outcome.

\section{SUPPORT STATEMENT}

Financial support was provided by Hospices Civils de Lyon

\section{STATEMENT OF INTEREST}

None declared.

\section{REFERENCES}

1 Brown KK. Rheumatoid lung disease. Proc Am Thorac Soc 2007; 4: 443-448.

2 Antoniou KM, Margaritopoulos G, Economidou F, et al. Pivotal clinical dilemmas in collagen vascular diseases associated with interstitial lung involvement. Eur Respir J 2009; 33: 882-896.

3 Bouros D, Pneumatikos I, Tzouvelekis A. Pleural involvement in systemic autoimmune disorders. Respiration 2008; 75: 361-371.

4 Devouassoux $\mathrm{G}$, Cottin V, Lioté $\mathrm{H}$, et al. Characterisation of severe obliterative bronchiolitis in rheumatoid arthritis. Eur Respir J 2009; 33: 1053-1061.

5 Turesson C, O'Fallon WM, Crowson CS, et al. Extra-articular disease manifestations in rheumatoid arthritis: incidence trends and risk factors over 46 years. Ann Rheum Dis 2003; 62: 722-727.

6 Freemer MM, King TE Jr. Connective tissue diseases. In: Schwarz MI, King TE Jr, eds. Interstitial lung disease. 4th Edn. Hamilton, B.C. Decker Inc, 2003: pp. 535-598.

7 Albano SA, Santana-Sahagun E, Weisman MH. Cigarette smoking and rheumatoid arthritis. Semin Arthritis Rheum 2001; 31: 146-159.

8 Klareskog L, Stolt P, Lundberg K, et al. A new model for an etiology of rheumatoid arthritis: smoking may trigger HLA-DR (shared epitope)-restricted immune reactions to autoantigens modified by citrullination. Arthritis Rheum 2006; 54: 38-46.

9 Kim EJ, Collard HR, King TE Jr. Rheumatoid arthritis-associated interstitial lung disease: the relevance of histopathologic and radiographic pattern. Chest 2009; 136: 1397-1405.

10 Kim DS. Interstitial lung disease in rheumatoid arthritis: recent advances. Curr Opin Pulm Med 2006; 12: 346-353.

11 Flaherty KR, Colby TV, Travis WD, et al. Fibroblastic foci in usual interstitial pneumonia: idiopathic versus collagen vascular disease. Am J Respir Crit Care Med 2003; 167: 1410-1415.

12 Song JW, Do KH, Kim MY, et al. Pathologic and radiologic differences between idiopathic and collagen vascular diseaserelated usual interstitial pneumonia. Chest 2009; 136: 23-30.

13 Rangel-Moreno J, Hartson L, Navarro C, et al. Inducible bronchusassociated lymphoid tissue (iBALT) in patients with pulmonary 
complications of rheumatoid arthritis. J Clin Invest 2006; 116: 3183-3194.

14 Park JH, Kim DS, Park IN, et al. Prognosis of fibrotic interstitial pneumonia: idiopathic versus collagen vascular disease-related subtypes. Am J Respir Crit Care Med 2007; 175: 705-711.

15 Gochuico BR, Avila NA, Chow CK, et al. Progressive preclinical interstitial lung disease in rheumatoid arthritis. Arch Intern Med 2008; 168: 159-166.

16 Lynch DA, David Godwin J, Safrin S, et al. High-resolution computed tomography in idiopathic pulmonary fibrosis: diagnosis and prognosis. Am J Respir Crit Care Med 2005; 172: 488-493.

17 Flaherty KR, Mumford JA, Murray S, et al. Prognostic implications of physiologic and radiographic changes in idiopathic interstitial pneumonia. Am J Respir Crit Care Med 2003; 168: 543-548.

18 Latsi PI, du Bois RM, Nicholson AG, et al. Fibrotic idiopathic interstitial pneumonia: the prognostic value of longitudinal functional trends. Am J Respir Crit Care Med 2003; 168: 531-537.

19 Hunninghake GW, Zimmerman MB, Schwartz DA, et al. Utility of a lung biopsy for the diagnosis of idiopathic pulmonary fibrosis. Am J Respir Crit Care Med 2001; 164: 193-196.

20 Raghu G, Mageto YN, Lockhart D, et al. The accuracy of the clinical diagnosis of new-onset idiopathic pulmonary fibrosis and other interstitial lung disease: A prospective study. Chest 1999; 116: $1168-1174$.

21 Elliot TL, Lynch DA, Newell JD Jr, et al. High-resolution computed tomography features of nonspecific interstitial pneumonia and usual interstitial pneumonia. J Comput Assist Tomogr 2005; 29: 339-345.

22 Akira M, Sakatani M, Hara H. Thin-section CT findings in rheumatoid arthritis-associated lung disease: CT patterns and their courses. J Comput Assist Tomogr 1999; 23: 941-948.

23 Kim EJ, Elicker BM, Maldonado F, et al. Usual interstitial pneumonia in rheumatoid arthritis-associated interstitial lung disease. Eur Respir J 2010; 35: 1322-1328.

24 Sokka T, Abelson B, Pincus T. Mortality in rheumatoid arthritis: 2008 update. Clin Exp Rheumatol 2008; 26: S35-S61.

25 Akira M, Inoue Y, Kitaichi M, et al. Usual interstitial pneumonia and nonspecific interstitial pneumonia with and without concurrent emphysema: thin-section CT findings. Radiology 2009; 251: 271-279.

26 Goh NS, Desai SR, Veeraraghavan S, et al. Interstitial lung disease in systemic sclerosis: a simple staging system. Am J Respir Crit Care Med 2008; 177: 1248-1254.

27 Ramos-Casals M, Brito-Zeron P, Munoz S, et al. Autoimmune diseases induced by TNF-targeted therapies: analysis of 233 cases. Medicine (Baltimore) 2007; 86: 242-251. 